\title{
Prevalencia de osteonecrosis de los maxilares asociada a medicamentos en pacientes tratados con bifosfonatos intravenosos: análisis epidemiológico en Centro del Cáncer - Red de Salud UC-CHRISTUS
}

\author{
Prevalence of medication-related osteonecrosis of the jaws in patients \\ treated with intravenous bisphosphonates: an epidemiologic analysis \\ at Centro del Cáncer - Red de Salud UC-CHRISTUS
}

\author{
Cristián Teuber L. ${ }^{1}$, Camila Foncea R. ${ }^{1}$, Francisco Rojas C. ${ }^{1}$, Kristine von Bischhoffshausen $P .{ }^{1}$, \\ Ignacio Goñi E. ${ }^{1}$, Alex Vargas D. ${ }^{1}$, Hernán Ramírez $S .{ }^{1}$
}

\section{Resumen}

Introducción: La osteonecrosis de los maxilares asociada a medicamentos (OMAM) es una patología que involucra la exposición necrótica de hueso maxilar o mandibular, relacionada al uso de fármacos antirresortivos y antiangiogénicos, con una prevalencia de $0,94 \%-13 \%$ en pacientes oncológicos y con osteoporosis que hacen uso de ellos. Objetivo: Determinar la prevalencia de osteonecrosis de los maxilares en pacientes en tratamiento con bifosfonatos intravenosos (BFIV) en el Centro del Cáncer de la Red de Salud UC-Christus, Santiago de Chile. Material y Método: Se analizaron los datos de pacientes que recibieron tratamiento de bifosfonatos intravenoso entre marzo y septiembre de 2016, con seguimiento por los equipos tratantes. Se consideró para la extracción de datos el género, edad, diagnóstico primario, bifosfonato intravenoso utilizado, tiempo de seguimiento, presencia de metástasis óseas y diagnóstico de OMAM. Resultados: Se obtuvo una muestra de 143 pacientes, con una relación hombre:mujer de 1:2; promedio de edad de 63,2 años; $78 \%$ de ellos fueron tratados con ácido zoledrónico y un $22 \%$ con pamidronato. Del total de pacientes un $1,4 \%(\mathrm{n}=2)$ desarrolló OMAM. Ambos casos con diagnóstico de cáncer de mama en tratamiento con ácido zoledrónico, lo que corresponde al 1,8\% de los pacientes en tratamiento con este fármaco. Conclusión: Si bien la OMAM es una patología infrecuente, esta se presenta con alta morbilidad y es de manejo complejo. La prevención y tratamiento de focos infecciosos odontogénicos de pacientes antes, durante o después del tratamiento con BFIV es fundamental para prevenir su desarrollo.

Palabras clave: bifosfonatos, ácido zoledrónico, pamidronato, osteonecrosis de los maxilares.

\footnotetext{
Abstract

Introduction: Medication-related osteonecrosis of the jaw (MRONJ) is a disease involving exposition of necrotic maxillary and mandibular bone and it's related to antiresorptive and antiangiogenic drugs, with a prevalence that variates from 0,94\%-13\% in oncologic and osteoporosis patients treated with them. Aim: To determine the prevalence of MRONJ in patients that underwent treatment with intravenous bisphosphonates (IVBP) at Centro del Cancer de la Red de Salud UC-CHRISTUS of Santiago, Chile. Material and Method: Data from patients who received intravenous bisphosphonate treatment between March and September 2016 were analyzed, with follow-ups by their treating teams. Data extraction considered gender, age, primary diagnosis, intravenous bisphosphonate used, follow up time, bone metastases and diagnosis of MRONJ. Results: A sample of 143 patients
}

'Departamento de Cirugía Oncológica y Maxilofacial, División de Cirugía, Facultad de Medicina Pontificia Universidad Católica de Chile. Santiago, Chile.

Los autores declaran no tener conflictos de interés.

Recibido el 6 de mayo de 2020. Aceptado el 29 de junio de 2020.

Correspondencia: Hernán Ramírez S. Departamento de Cirugía Oncológica y Maxilofacial, División de Cirugía, Facultad de Medicina,

Pontificia Universidad Católica de Chile.

Av. Diagonal Paraguay 362, $3^{\circ}$ Piso. Santiago, Chile. Email: hramirez.skinner@ gmail.com 
was obtained with a men:women ratio of 1:2; an average age of 63,2 years, $78 \%$ of the patients were treated with zoledronic acid and $22 \%$ of the patients with pamidronate. From the total number of patients, $1.4 \%(n=2)$ developed MRONJ, both cases had breast cancer as primary diagnosis and in treatment with zoledronic acid, which corresponds to $1.8 \%$ of patients being treated with this drug. Conclusion: Although MRONJ is an infrequent disease, it presents high morbidity and complex management. Prevention and treatment of odontogenic infectious foci in patients before, during and after treatment with IVBP drugs is fundamental to prevent this pathology.

Keywords: bisphosphonates, zoledronic acid, pamidronate, osteonecrosis of the jaw.

\section{Introducción}

La osteonecrosis de los maxilares asociada a medicamentos (OMAM), definida por la American Association of Oral and Maxillofacial Surgeons (AAOMS), se basa en tres criterios simultáneos: 1) presencia de hueso necrótico expuesto o que puede ser explorado mediante una fístula en la región maxilofacial, y que se mantiene durante un periodo mínimo de ocho semanas; 2) pacientes que han sido tratados con fármacos antirresortivos o antiangiogénicos; 3 ) pacientes que no hayan sido tratados con radioterapia o presenten metástasis óseas maxilofaciales evidentes ${ }^{1-3}$.

Los fármacos antirresortivos alteran el metabolismo óseo afectando directamente la actividad de los osteoclastos. Estas drogas se han convertido en la terapia de elección para desórdenes esqueletales caracterizados por un aumento de la reabsorción ósea, como la osteoporosis, enfermedad de Paget, osteogénesis imperfecta, hipercalcemia maligna, metástasis óseas de tumores sólidos y mieloma múltiple. Fármacos como los bifosfonatos y denosumab se incluyen en este grupo de medicamentos ${ }^{1,4,5}$. Este último corresponde a un anticuerpo monoclonal que actúa sobre los receptores activadores del factor nuclear kappa B ligando (RANK-L), un componente clave en la formación y activación de los osteoclastos ${ }^{4-6}$.

Los bifosfonatos administrados por vía intravenosa (BFIV), como por ejemplo el ácido zoledrónico, pamidronato y alendronato, se utilizan habitualmente como parte del tratamiento de la osteopenia grave, metástasis óseas e hipercalcemia severa. Se ha establecido que la terapia con bifosfonatos en estos casos, reduce el número de fracturas, mejora la morfología del hueso y disminuye el dolor. Por otra parte, los bifosfonatos por vía oral se indican principalmente para el tratamiento de osteoporosis y osteopenia, aunque desde el año 2007 Food and Drug Administration (FDA) aprueba el uso de ácido zoledrónico en estos pacientes ${ }^{4,6,7}$.

En cuanto a los fármacos antiangiogénicos, estos son utilizados en el tratamiento de tumores gastrointestinales, neuroendocrinos, carcinomas renales y otras enfermedades malignas. Estos actúan inhibiendo la formación de vasos sanguíneos e interrumpiendo su señalización. Sunitinib o bevacizumab son ejemplos de estos ${ }^{1}$. Sunitinib inhibe las señales celulares por unión a múltiples receptores de tirosina quinasa, incluyendo los receptores de factor de crecimiento derivado de plaquetas y receptores del factor de crecimiento endotelial, los que actúan en la angiogénesis y la proliferación de células tumorales. Por su parte el bevacizumab corresponde a un anticuerpo monoclonal que se une al factor de crecimiento endotelial vascular, inhibiendo la señalización de la angiogénesis ${ }^{1,2}$.

Ambos grupos farmacológicos han demostrado ser efectivos, pero desde el año 2003 se comenzó a reportar casos de osteonecrosis de los maxilares en pacientes que se encontraban, o tenían historia de haber recibido tratamiento con estos medicamentos ${ }^{1-6}$. Respecto a este tema, la mayoría de los autores señalan a los BFIV como los fármacos antirresortivos que se han visto más frecuentemente asociados al desarrollo de $\mathrm{MRONJ}^{1-6}$.

\section{Objetivo}

El objetivo de este trabajo es realizar un análisis descriptivo, retrospectivo, de los pacientes que fueron tratados con BFIV en el Centro del Cáncer de la Red de Salud UC-CHRISTUS de Santiago de Chile, con el fin de evaluar la frecuencia de OMAM en este grupo y comparar nuestra experiencia con la literatura. 


\section{Material y Método}

Estudio retrospectivo, descriptivo, de 143 pacientes que se encontraban en tratamiento con BFIV en el Centro del Cáncer de la Red de Salud UC-CHRISTUS, Santiago de Chile, durante marzo a septiembre del año 2016. En los datos obtenidos se consideraron las variables de género, edad, diagnóstico primario, bifosfonato intravenoso utilizado, metástasis óseas, tiempo de seguimiento y diagnóstico de OMAM. Para los criterios de inclusión se consideró a todos los pacientes que estuvieran en tratamiento con BFIV durante el periodo de marzo a septiembre de 2016, con registros médicos completos. Los criterios de exclusión se aplicaron a pacientes que hubieran recibido radioterapia de cabeza y cuello y/o fármacos antirresortivos/antiangiogénicos y casos con presencia de metástasis óseas maxilares o mandibulares.

La información se obtuvo a partir de la base de datos y fichas clínicas de cada paciente, siguiendo registros clínicos de posibles cuadros de OMAM hasta su último control con su equipo tratante. El trabajo fue aprobado por el Comité de Ética de la Facultad de Medicina de la Pontificia Universidad Católica de Chile. El ingreso y análisis de datos se realizó a través del programa Epi Info, con anonimización de los datos personales de los pacientes, y confección de tablas de frecuencia con análisis por rango etario, diagnóstico primario, BFIV utilizado y presencia de metástasis óseas (versión 7.0, CDC, Atlanta, USA).

\section{Resultados}

De un total de 143 pacientes tratados con BFIV, el 33,6\% $(\mathrm{n}=48)$ eran hombres y el $66,4 \%(\mathrm{n}=95)$ mujeres, con una relación de hombre a mujer de 1:2. La edad media fue de 63,2 años (con una desviación estándar de 14,27 ), oscilando entre 21 y 92 años. El 28,7\% $(\mathrm{n}=41)$ de los pacientes tenían entre 60 y 69 años y el $25,3 \%(n=36)$ entre 70 y 80 años (Tabla 1).

El promedio de seguimiento fue de 19,62 meses (desde 1 hasta 100 meses posadministración de la última dosis de BFIV). Del total de pacientes, un $78 \%(\mathrm{n}=111)$ fue tratado con ácido zoledrónico, mientras que un $22 \%$ $(\mathrm{n}=32)$ recibió tratamiento intravenoso con pamidronato. De los pacientes que cumplieron los criterios de inclusión y exclusión, el 30,8\% ( $\mathrm{n}=44)$ tuvo como diagnóstico primario cáncer de mama, el 28\% $(\mathrm{n}=32)$ mieloma múltiple y el $18,2 \%(\mathrm{n}=24)$ cáncer de próstata. En el caso de los pacientes con cáncer de mama, la terapia antirresortiva fue indicada en el contexto del manejo de metástasis óseas en $88,6 \%$ de los casos y por osteopenia severa en el $11,4 \%$. Para los pacientes con cáncer de próstata, la terapia fue indicada debido a metástasis en un 92,3\% de los casos y por os-

\begin{tabular}{|c|c|c|c|c|c|c|}
\hline \multirow[t]{2}{*}{ Grupo etario } & \multicolumn{2}{|c|}{ Femenino } & \multicolumn{2}{|c|}{ Masculino } & \multicolumn{2}{|c|}{ Total } \\
\hline & n & $\%$ & $\mathrm{n}$ & $\%$ & $\mathbf{n}$ & $\%$ \\
\hline $20-29$ & 1 & 1,05 & 1 & 2,08 & 2 & 1,39 \\
\hline $30-39$ & 8 & 8,42 & 0 & 0 & 8 & 5,59 \\
\hline $40-49$ & 8 & 8,42 & 4 & 8,33 & 12 & 8,39 \\
\hline $50-59$ & 24 & 25,26 & 6 & 12,5 & 30 & 20,98 \\
\hline $60-69$ & 31 & 32,63 & 10 & 20,83 & 41 & 28,67 \\
\hline $70-80$ & 15 & 15,78 & 21 & 43,75 & 36 & 25,17 \\
\hline $81+$ & 8 & 8,42 & 6 & 12,5 & 14 & 9,79 \\
\hline Total & 95 & 100 & 48 & 100 & 143 & 100 \\
\hline
\end{tabular}




\section{Tabla 2. Distribución por diagnóstico primario y BFIV utilizado como tratamiento}

\begin{tabular}{|c|c|c|c|c|c|c|c|c|}
\hline \multirow[b]{2}{*}{ Diagnóstico } & \multicolumn{2}{|c|}{ Total de casos } & \multicolumn{2}{|c|}{ Metástasis óseas } & \multirow[b]{2}{*}{$\mathbf{n}$} & \multirow[b]{2}{*}{$\%$} & \multicolumn{2}{|c|}{ Pamidronato } \\
\hline & $\mathbf{n}$ & $\%$ & $\mathbf{n}$ & $\%$ & & & $\mathbf{n}$ & $\%$ \\
\hline Cáncer de mama & 44 & 30,8 & 39 & 88,6 & 41 & 28,7 & 3 & 2,1 \\
\hline Mieloma múltiple & 40 & 28,0 & - & - & 19 & 13,3 & 2 & 14,7 \\
\hline Cáncer de próstata & 26 & 18,2 & 24 & 92,3 & 22 & 15,4 & 4 & 2,8 \\
\hline Osteoporosis & 16 & 11,2 & - & - & 15 & 10,5 & 1 & 0,7 \\
\hline Cáncer de pulmón & 13 & 9,1 & 13 & 100,0 & 11 & 7,7 & 2 & 1,4 \\
\hline Cáncer de colon & 3 & 2,1 & 3 & 100,0 & 2 & 1,4 & 1 & 0,7 \\
\hline Cáncer gástrico & 1 & 0,7 & 1 & 100,0 & 1 & 0,7 & 0 & 0,0 \\
\hline Total & 143 & 100,0 & 80 & 91,95 & 111 & 77,6 & 32 & 22,4 \\
\hline
\end{tabular}

teopenia grave en 7,7\%. La Tabla 2 resume la distribución de los pacientes según diagnóstico y tipo de BFIV utilizado.

Del total de pacientes tratados con BFIV en el periodo de tiempo señalado, sólo dos (1,4\% de los casos) presentaron OMAM. Los dos casos fueron pacientes en tratamiento antirresortivo con ácido zoledrónico debido a un cáncer de mama con metástasis óseas. Los pacientes llevaban en tratamiento con BFIV 4 y 35 meses respectivamente. Cuando se analizó el total de pacientes en tratamiento con ácido zoledrónico en ese periodo de tiempo, la incidencia de MRONJ para este grupo fue del $1,8 \%$.

\section{Discusión}

Los bifosfonatos son fármacos antirresortivos análogos del pirofosfato, que actúan a nivel óseo comportándose como inhibidores naturales de los osteoclastos. Cuando los bifosfonatos se incorporan a los osteoclastos, se desencadena su apoptosis, alterando así el ciclo normal del tejido óseo, que tiene como consecuencia: inhibición de la resorción ósea, disminución en el recambio óseo y aumento de la densidad ósea ${ }^{8}$. El efecto de los bifosfonatos persiste durante el tiempo, con una vida media para los administrados vía intravenosa, en promedio 11,2 años ${ }^{3-6}$. Este efecto, se debe a una alta afinidad sumada a la unión irreversible con los cristales de hidroxiapatita que forman parte del tejido óseo. El efecto ocurre principalmente en el osteoclasto, alterando su citoesqueleto, estimulando su apoptosis y reduciendo la expresión de bombas de protones ${ }^{6-10}$.

Los resultados obtenidos en este estudio se corresponden con los datos disponibles en la literatura. Ibrahim y cols. (2006) publican un estudio retrospectivo que incluye 539 pacientes en tratamiento con BFIV por metástasis óseas de tumores sólidos, donde el $58 \%$ de los pacientes incluidos eran mujeres y el $42 \%$ hombres, con una edad promedio de 64 años; dando una relación hombre:mujer de 1:1.59. En nuestro estudio se observó que el $77 \%$ de los pacientes se encontraba en tratamiento con ácido zoledrónico y el $23 \%$ con pamidronato, en comparación con lo descrito por Ibrahim (2006) donde el 95,5\% recibió tratamiento con ácido zoledrónico y solo el 1,5\% con pamidronato9. Es probable que un mayor porcentaje de pacientes sean tratados con ácido zoledrónico por sobre otras alternativas, debido a que corresponde a un bifosfonato de tercera generación, 100 veces más potente que el pamidronato ${ }^{10}$. Sumado a lo anterior, se ha demostrado que el ácido zoledrónico inhibe de forma efectiva el crecimiento, migración e invasión de células cancerígenas en el hueso ${ }^{11}$.

Para los casos incluidos en el estudio, se observó que más del 75\% de los pacientes que fueron tratados con BFIV presentaron como diagnóstico primario cáncer de mama, mieloma múltiple o cáncer de próstata, lo que también se correlaciona con los datos disponibles 
en la literatura ${ }^{12}$. Para este grupo de pacientes, sobre todo en caso de metástasis óseas, la terapia antirresortiva permite disminuir el dolor y la morbilidad esqueletal, mejorando la calidad de vida y la tasa de supervivencia ${ }^{13}$.

Si bien la OMAM es infrecuente, puede asociarse a una morbilidad importante y afectar muy negativamente la calidad de vida ${ }^{14,15}$. Las tasas de frecuencia observadas fluctúan entre el $0,94 \%$ al $13 \%$ en diferentes grupos de población con diferentes regímenes de drogas ${ }^{16,17}$. Para el caso específico de pacientes tratados con ácido zoledrónico, se ha descrito una frecuencia cercana al $1,3 \%^{18}$. Se plantea que las osteonecrosis en pacientes en tratamiento con bifosfonatos podría desencadenarse debido a su efecto sobre los osteoclastos y la inhibición de la remodelación ósea, lo que asociado a la cicatrización inadecuada de los tejidos en caso de una injuria, patologías de base y tratamiento con otros fármacos, podría facilitar una necrosis avascular e infección por pató- genos $^{19-21}$. Dentro de los factores etiológicos que se han asociado al desarrollo de OMAM, las exodoncias y los procedimientos odontológicos invasivos dentoalveolares son los más frecuentemente relacionados ${ }^{16,17}$.

Para los dos casos que presentaron OMAM en el grupo de pacientes estudiados, el factor desencadenante fue la exodoncia. Ambos debutaron con síntomas inespecíficos como dolor persistente e inflamación local en la zona intervenida. En ambos casos, a pesar de los cuidados locales y el uso de antibióticos sistémicos cuando fue necesario, los pacientes requirieron ser sometidos a cirugía bajo anestesia general para aseo quirúrgico y resección de secuestros óseos secundarios a la progresión de la necrosis ósea (Figuras 1, 2 y 3 ).

La frecuencia de OMAM observada en este estudio coincide con lo descrito en la literatura. En su estudio, Sven y cols. (2018) concluyeron que las bajas tasas de frecuencia (1,3\% para pacientes bajo ácido zoledrónico) de OMAM

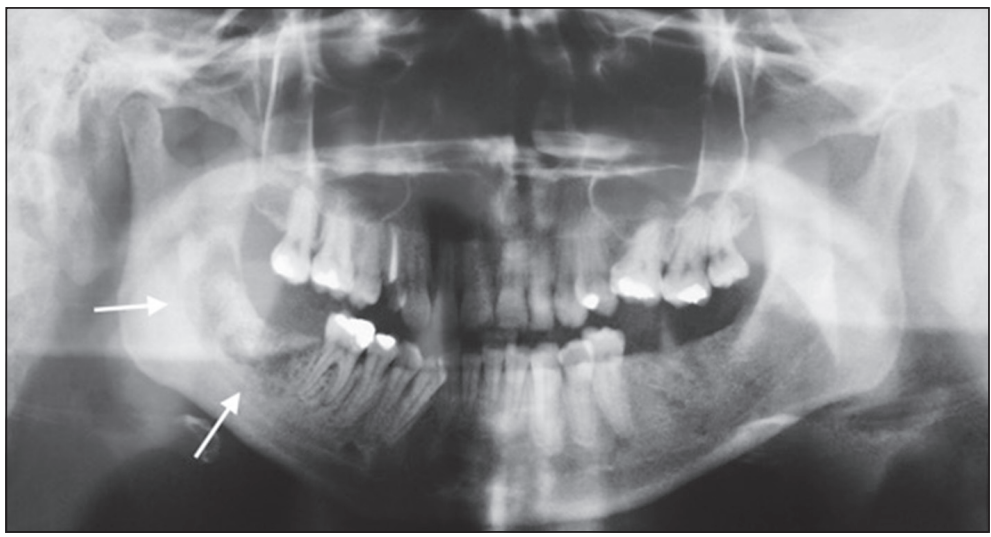

Figura 1. Radiografía panorámica con osteonecrosis en ángulo mandibular derecho (fecha blanca). Paciente en tratamiento con bifosfonato intravenoso.

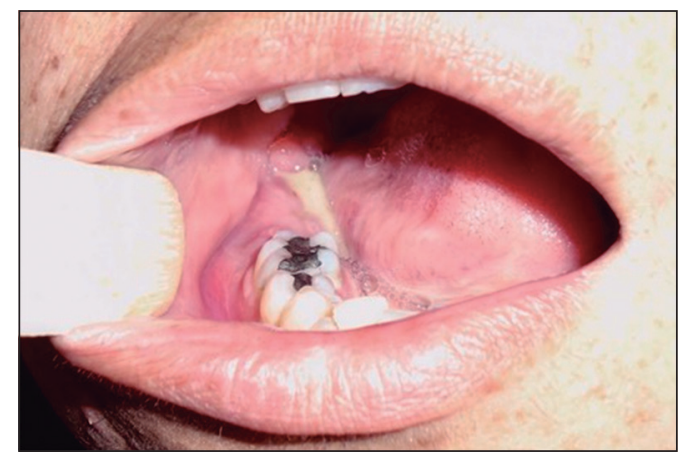

Figura 2. Fístula mandibular derecha con supuración activa.

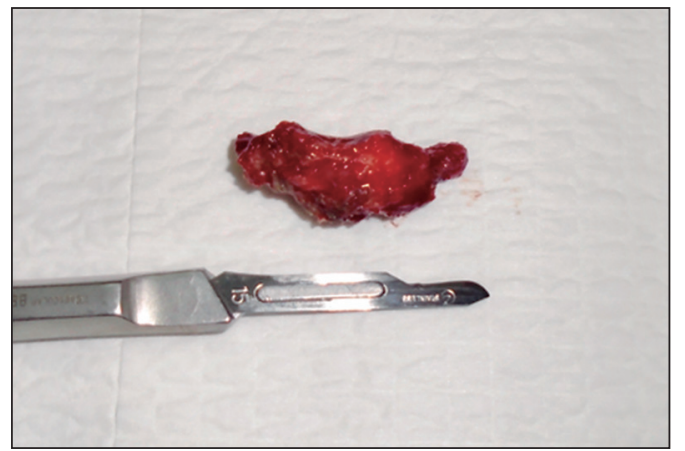

Figura 3. Secuestro óseo mandibular derecho resecado. 


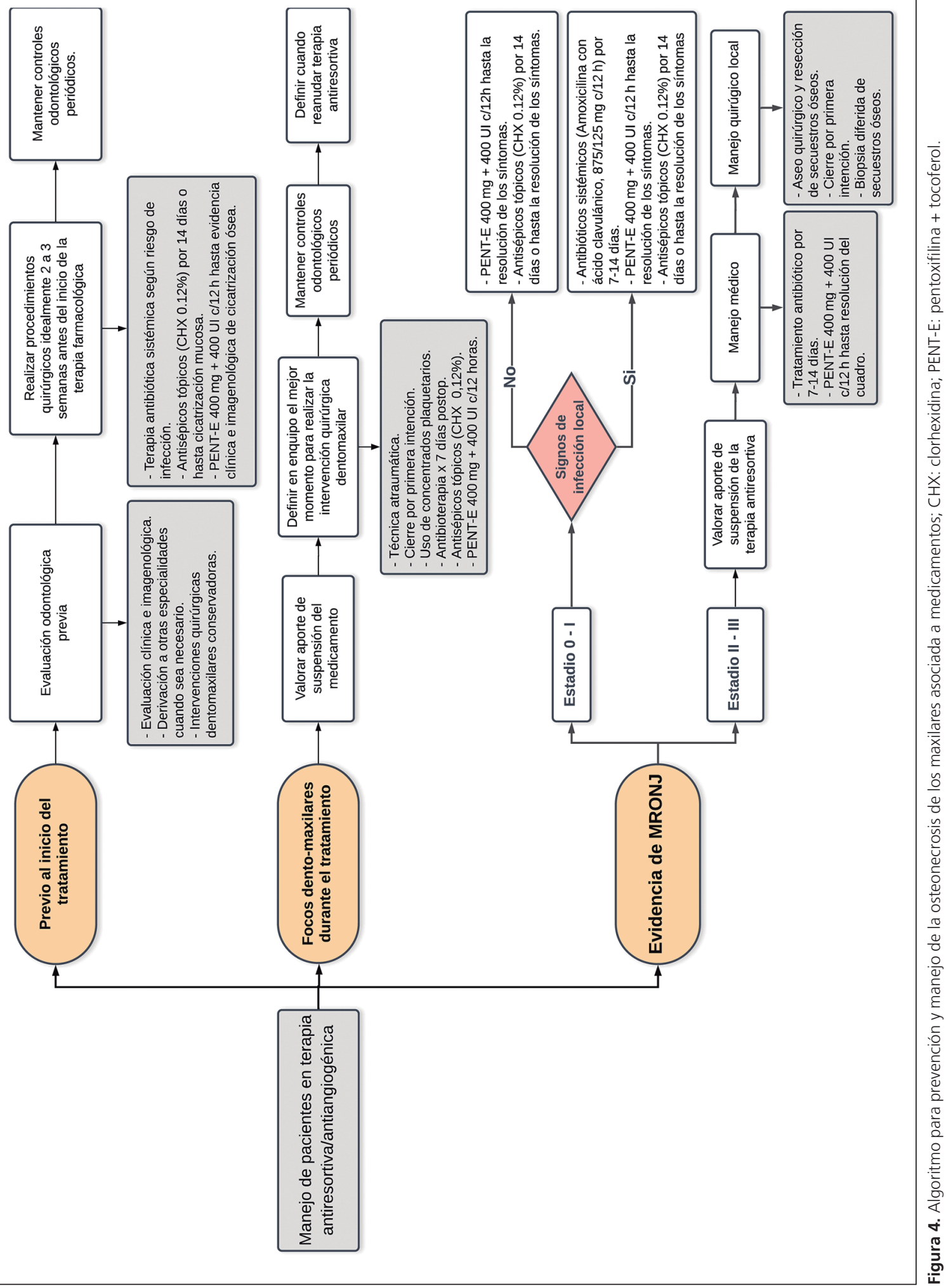


se deben a un riguroso protocolo previo al inicio de la terapia antirresortiva ${ }^{18}$. Si bien la OMAM se puede producir de forma espontánea, la mayoría de los casos presenta como factor desencadenante común una exodoncia y/o procedimientos dentales invasivos ${ }^{22,23}$. Es fundamental que todo paciente reciba una rigurosa evaluación de su salud oral previo a que se inicie el tratamiento con fármacos antirresortivos $^{23,24}$. Por lo anteriormente planteado, sumado a nuestra experiencia y la literatura relacionada, todo paciente que requiere iniciar tratamiento con BFIV en nuestra institución debe ser evaluado previamente de forma interdisciplinaria. En conjunto a los equipos de Cirugía Oncológica y Maxilofacial, y Oncología Médica de la Red de Salud UC-CHRISTUS, se desarrolló un protocolo para la prevención y manejo de esta patología, el cual se detalla en la Figura $4^{23}$.

En este protocolo se consideran las medidas preventivas a considerar en estos pacientes, donde es fundamental que todos sean evaluados por medio de un examen clínico e imagenológico por un cirujano maxilofacial o bien un odontólogo con experiencia, con el objetivo de definir la necesidad de realizar procedimientos quirúrgicos invasivos (como por ejemplo, exodoncias) previo al inicio del tratamiento.

\section{Conclusión}

Si bien la incidencia de la OMAM en pacientes en tratamiento con BFIV es baja, y puede tardar en desarrollarse en promedio 4 años, es fundamental establecer adecuadas estrategias para minimizar su riesgo de aparición. La OMAM puede asociarse a una morbilidad importante, la cual se agrega a las patologías de base que motivan el tratamiento con BFIV en primera instancia.

Como parte de estas estrategias de prevención, es fundamental la evaluación y tratamiento oportuno de los focos infecciosos odontogénicos antes de iniciar el tratamiento con BFIV, lo cual es clave para minimizar la frecuencia de estos casos. En nuestro centro y gracias a un trabajo interdisciplinario, hemos implementado un riguroso protocolo clínico e imagenológico que ha favorecido a mantener una baja prevalencia de esta complicación, sin embargo, son necesarios nuevos estudios para lograr un mejor entendimiento de esta patología, incluyendo el análisis de fármacos inhibidores de RANK-L, receptores de tirosina quinasa y antiangiogénicos.

\section{Bibliografía}

1. Ruggiero S, Dodson T, Fantasia C, et al. American association of oral and maxillofacial surgeons position paper on medication-related osteonecrosis of the jaw - 2014 update. J Oral Maxillofac Surg. 2014;10:1938-56 doi:10.1016/j. joms.2014.04.031.

2. Ruggiero S. Diagnosis and Staging of MedicationRelated Osteonecrosis of the Jaw. Oral Maxillofac Surg Clin North Am. 2015;27(4):479-487. doi:10.1016/j. coms.2015.06.008.

3. Khan A, Morrison A, Hanley D, et al. Diagnosis and management of osteonecrosis of the jaw: A systematic review and international consensus. Journal of Bone and Mineral Research. 2015;30(1):3-23. doi:10.1002/ jbmr.2405.

4. Fleisher K, Kontio R, Otto S. Antiresorptive DrugRelated Osteonecrosis of the Jaw (ARONJ)-a Guide to Research. Thieme 2016. AO Foundation, Davos Platz, Switzerland.

5. Russell RG. Bisphosphonates: mode of action and pharmacology. Pediatrics. 2007;119 Suppl 2:S150-S162. doi:10.1542/peds.2006-2023H.

6. Aghaloo T, Dry S, Mallya S, Tetradis S. Stage 0 osteonecrosis of the jaw in a patient on denosumab. J Oral Maxillofac Surg 2014;72(4):702-16. doi:10.1016/j.joms.2013.09.008.

7. Sharma D, Ivanovski S, Slevin M, et al. Bisphosphonate-related osteonecrosis of jaw (BRONJ): Diagnostic criteria and possible pathogenic mechanisms of an unexpected anti-angiogenic side effect. Vasc Cell. 2013; 5:1. doi:10.1186/2045824X-5-1.

8. Sawatari Y, Marx R. Bisphosphonates and Bisphosphonate Induced Osteonecrosis. Oral Maxillofac Surg Clin North Am. 2007;19(4):487-498. doi:10.1016/j.coms.2007.07.003.

9. McClung M, Harris S, Miller P, et al. Bisphosphonate therapy for osteoporosis: benefits, risks, and drug holiday. Am J Med. 2013;126(1):13-20. doi: 10.1016/j. amjmed.2012.06.023.

10. Ibrahim T, Barbanti F, Giorgio-Marrano G, et al. Osteonecrosis of the jaw in patients with bone metastases treated with bisphosphonates: a retrospective study. Oncologist. 2008;13(3):330-336. doi:10.1634/theoncologist.2007-0159.

11. Wellington K, Goa K. Zoledronic acid: A review of its use in the management of bone metastases 
and hypercalcaemia of malignancy. Drugs. 2003;63(4):417-437. doi:10.2165/00003495200363040-00009.

12. Dedes P, Gialeli C, Tsonis A, et al. Expression of matrix macromolecules and functional properties of breast cancer cells are modulated by the bisphosphonate zoledronic acid. Biochim Biophys Acta-Gen Subj. 2012;1820(12):1926-1939. doi:10.1016/j.bbagen.2012.07.013.

13. Stopeck A, Fizazi K, Body J, et al. Safety of long-term denosumab therapy: results from the open label extension phase of two phase 3 studies in patients with metastatic breast and prostate cancer. Support Care Cancer. 2016;24(1):447-455. doi: 10.1007/ s00520-015-2904-5.

14. Tesfamariam Y, Jakob T, Wöckel A, et al. Adjuvant bisphosphonates or RANK-ligand inhibitors for patients with breast cancer and bone metastases: A systematic review and network meta-analysis. Crit Rev Oncol Hematol. 2019;137:1-8. doi:https://doi. org/10.1016/j.critrevonc.2019.02.004.

15. Hoff A, Toth B, Altundag K, et al. Frequency and risk factors associated with osteonecrosis of the jaw in cancer patients treated with intravenous bisphosphonates. J Bone Miner Res. 2008;23: 826-836 doi:10.1359/jbmr.080205.

16. Ruggiero S, Dodson T, Assael L, Landesberg R, Marx R, Mehrotra B. American Association of Oral and Maxillofacial Surgeons Position Paper on Bisphosphonate-Related Osteonecrosis of the Jaw - 2009 update. Aust Endod J. 2009; 35:119-130. doi:10.1111/j.1747-4477.2009.00213.x.

17. McLeod N, Brennan P, Ruggiero S. Bisphosphonate osteonecrosis of the jaw: a historical and contemporary review. Surg. 2012;10(1):36-42. doi: 10.1016/j.surge.2011.09.002.

18. Hellstein JW, Adler RA, Edwards B, et al. Managing the care of patients receiving antiresorptive therapy for prevention and treatment of osteoporosis: executive summary of recommendations from the American Dental Association Council on Scientific Affairs. J Am Dent Assoc. 2011;142(11):1243-1251.

19. Otto S, Pautke C, Van den Wyngaert T, Niepel D, Schiødt M. Medication-related osteonecrosis of the jaw: Prevention, diagnosis and management in patients with cancer and bone metastases. Cancer Treat Rev. 2018;69:177-187. doi: 10.1016/j. ctrv.2018.06.007.

20. Otto S, Hafner S, Mast G, et al. BisphosphonateRelated Osteonecrosis of the Jaw: Is pH the Missing Part in the Pathogenesis Puzzle? J Oral Maxillofac Surg. 2010;68(5):1158-1161. doi:10.1016/j. joms.2009.07.079.

21. Yamashita J, McCauley L, Van Poznak C. Updates on osteonecrosis of the jaw. Curr Opin Support Palliat Care. 2010;4(3):200-6. doi:10.1097/ SPC.0b013e32833d303b.

22. Saad F, Brown J, van Poznak C, et al. Incidence, risk factors, and outcomes of osteonecrosis of the jaw: integrated analysis from three blinded activecontrolled phase III trials in cancer patients with bone metastases. Ann Oncol. 2012; 23(5):1341-7. doi: 10.1093/annonc/mdr435.

23. Foncea C, Von Bischhoffshausen K, Teuber C, et al. Osteonecrosis de los maxilares asociada a medicamentos: Revisión de la literatura y propuesta para la prevención y manejo. Rev Med Chile. (en prensa) 2019.

24. Aljohani S, Fliefel R, Ihbe J, Kühnisch J, Ehrenfeld $\mathrm{M}$, Otto $\mathrm{S}$. What is the effect of anti-resorptive drugs (ARDs) on the development of medication-related osteonecrosis of the jaw (MRONJ) in osteoporosis patients: A systematic review. J Craniomaxillofac Surg. 2017;45(9):1493-1502. doi:10.1016/j. jcms.2017.05.028.

25. Vanpoecke J, Verstraete L, Smeets M, Ferri J, Nicot $\mathrm{R}$ y Politis C. Medication-related osteonecrosis of the jaw (MRONJ) stage III: Conservative and conservative surgical approaches versus an aggressive surgical intervention: A systematic review. J Craniomaxillofac Surg. 2020;48(4):435-443. doi:10.1016/j.jcms.2020.02.017.

26. Klingelhöffer C, Zeman F, Meier J, Reichert TE, Ettl T. Evaluation of surgical outcome and influencing risk factors in patients with medication-related osteonecrosis of the jaws. J Craniomaxillofac Surg. 2016;44(10):1694-1699. doi:10.1016/j. jcms.2016.08.001. 